\title{
Towards the development of a compact-monolithic optical assembly for Telecom laser frequency stabilization for LISA ground tests and other applications
}

Ouali Acef, Alexis Mehlman, David Holleville, Michel Lours, Rodolphe Le Targat, et al.

Ouali Acef, Alexis Mehlman, David Holleville, Michel Lours, Rodolphe Le Targat, Peter Wolf, Henri Lehec, Sébastien Bize, Ouali Acef, Aurelien Boutin, Karine LePage, Ludovic Fulop, Laure Oudda, Thomas Lévèque, "Towards the development of a compact-monolithic optical assembly for Telecom laser frequency stabilization for LISA ground tests and other applications," Proc. SPIE 11852, International Conference on Space Optics - ICSO 2020, 118523P (11 June 2021); doi: 10.1117/12.2599617

SPIE Event: International Conference on Space Optics - ICSO 2021, 2021, Online Only 


\section{International Conference on Space Optics-ICSO 2020}

Virtual Conference

30 March-2 April 2021

Edited by Bruno Cugny, Zoran Sodnik, and Nikos Karafolas
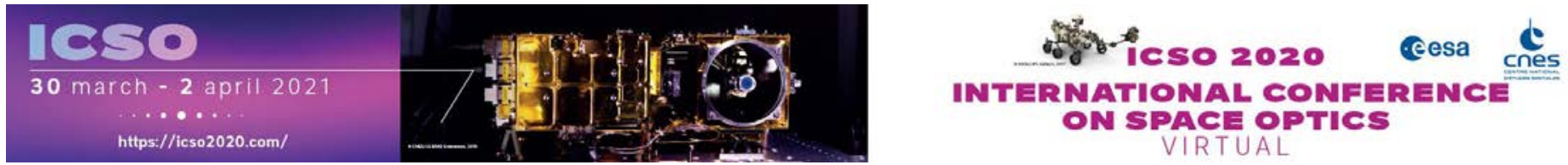

\section{Towards the development of a compact-monolithic optical assembly for Telecom laser frequency stabilization for LISA ground tests and other applications}

\section{Cesa issopoeseatings lenes}




\title{
Towards the development of a compact-monolithic optical assembly for Telecom laser frequency stabilization for LISA ground tests and other applications
}

\author{
Alexis Mehlman ${ }^{1,2}$, David Holleville ${ }^{1}$, Michel Lours ${ }^{1}$, Rodolphe Le Targat ${ }^{1}$, \\ Peter Wolf ${ }^{1}$, Henri Lehec ${ }^{1}$, Sébastien Bize ${ }^{1}$, Ouali Acef ${ }^{1}$, Aurélien Boutin ${ }^{2}$, \\ Karine Lepage ${ }^{2}$, Ludovic Fulop ${ }^{2}$, Laure Oudda ${ }^{3}$, Thomas Lévèque ${ }^{3}$ \\ ${ }^{1}$ LNE-SYRTE, Observatoire de Paris-Université PSL, CNRS, Sorbonne Université \\ 61 avenue de l'Observatoire, F-75014 Paris, France. \\ ${ }^{2}$ KYLIA, 10 rue de Montmorency, F-75003 Paris France. \\ ${ }^{3}$ CNES, 18 Avenue Edouard Belin 31401, Toulouse Cedex 9, France.
}

\begin{abstract}
We report on an ongoing development of a compact and transportable iodine frequency stabilized laser setup, based on compact and fibered Telecom components with a high technological readiness level (TRL). This laser system is being planned as a compact and easily transportable ultra-stable frequency reference for ground tests of the payload of LISA mission (Laser Interferometer Space Antenna) as part of the SYRTE laboratory contribution to the French activities carried out by a consortium of several partners lead by the French Space Agency (CNES) for assembly-integration and tests (AIVT) of the payload of LISA. This frequency reference will match the LISA requirements in terms of both residual frequency and intensity noise. The target residual frequency noise for this stabilized laser is below the nominal requirement for LISA, which is currently $30 \mathrm{~Hz} / \sqrt{ } \mathrm{Hz}$. Thus, we propose to provide a tandem of $1064 \mathrm{~nm}$ laser sources phase-locked to an iodine stabilized Telecom laser operating at $1596 \mathrm{~nm}$. The frequency gap between the telecom domain and the green range is bridged using a frequency tripling process based on two cascaded PPLN crystals, fully fibered. The compact design of the whole setup will make it easily transportable to different sites and could be readily used for ground tests of the LISA payload.
\end{abstract}

Keywords: LISA space mission, Frequency stabilized laser, Iodine vapor, PPLN crystals, third harmonic generation

\section{INTRODUCTION}

Ultra-stable lasers provided in compact optical architecture are an important technological key for many embedded applications, such as gravitational wave detection in space, earth observations/gravity field mapping, ground to space or inter-satellites optical links, accurate laser ranging [1-3], etc... Nowadays, the requirements in terms of residual laser frequency noise, expressed in terms of Allan deviation, for many of these applications are in the range of $10^{-14}-10^{-15}$, easily reached in a laboratory environment [4-6].

Our development is based on fibered telecom photonic components associated with a compact-monolithic spectroscopy optical bench. We take advantage of an existing laser frequency stabilization experiment based on a Telecom laser diode at $1544 \mathrm{~nm}$, frequency tripled and stabilized against a narrow iodine line at $\sim 514.6 \mathrm{~nm}$ [7]. The residual frequency noise already achieved for this experiment is below the LISA mission requirements. We achieve a reproducible frequency stability below $2.5 \times 10^{-14} \tau^{-1 / 2}$ decreasing to $3.5 \times 10^{-15}$ level after $200 \mathrm{~s}$ of integration time. It corresponds to an amplitude spectral density of the frequency fluctuations $15 \mathrm{~Hz} / \sqrt{ } \mathrm{Hz}$. Furthermore, we demonstrated the ability to transfer the frequency stability achieved around $1.5 \mu \mathrm{m}$ to the near infrared range, close to $1 \mu \mathrm{m}$, in a simple manner, using the usual phase locking loop technique associated to a second harmonic generation process [8]. 


\section{LASER DESCRIPTION}

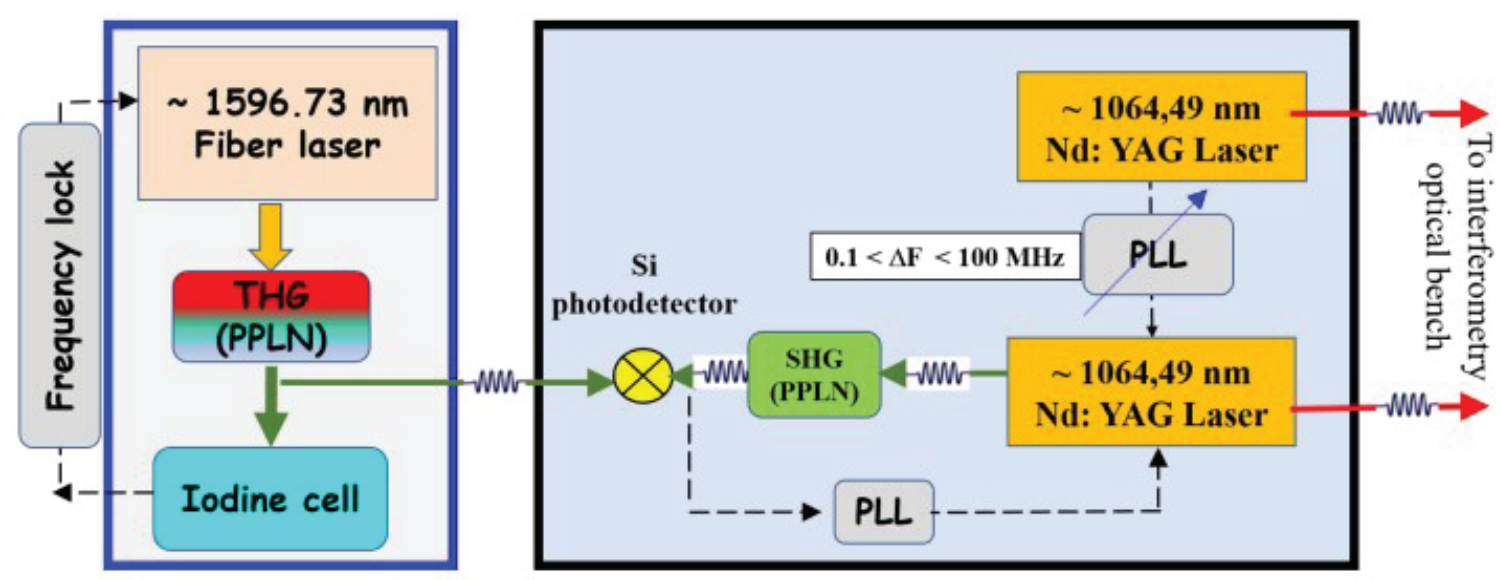

Figure 1. Whole frequency stabilized laser ensemble under development for the ground tests of LISA

Figure 1 describes the laser ensemble under development for the ground tests of LISA. It is made of two distinct parts. The first one (right part in the black rectangle) consists of two $1064 \mathrm{~nm}$ lasers, phase-locked with respect to each other, with a tunable frequency offset -with a rate up to $10 \mathrm{kHz} / \mathrm{s}-$ over the entire LISA frequency band. The phase lock bandwidth is $>34 \mathrm{kHz}$, and the contribution of the residual frequency noise of the PLL is significantly below the LISA frequency noise requirement. The RIN of the two phase-locked $1064 \mathrm{~nm}$ lasers is already below $10^{-7} / \sqrt{ } \mathrm{Hz}$ level, at the LISA heterodyne frequency, limited by the detection noise. The first part is already completed, tested and ready for delivery to test/integration laboratories. The laser setup will be used for accurate testing of interferometric benches mechanical stability needed for the MOSA-LISA AIVT, with the same level -or better- than the LISA mission requirements in terms of residual frequency instabilities.
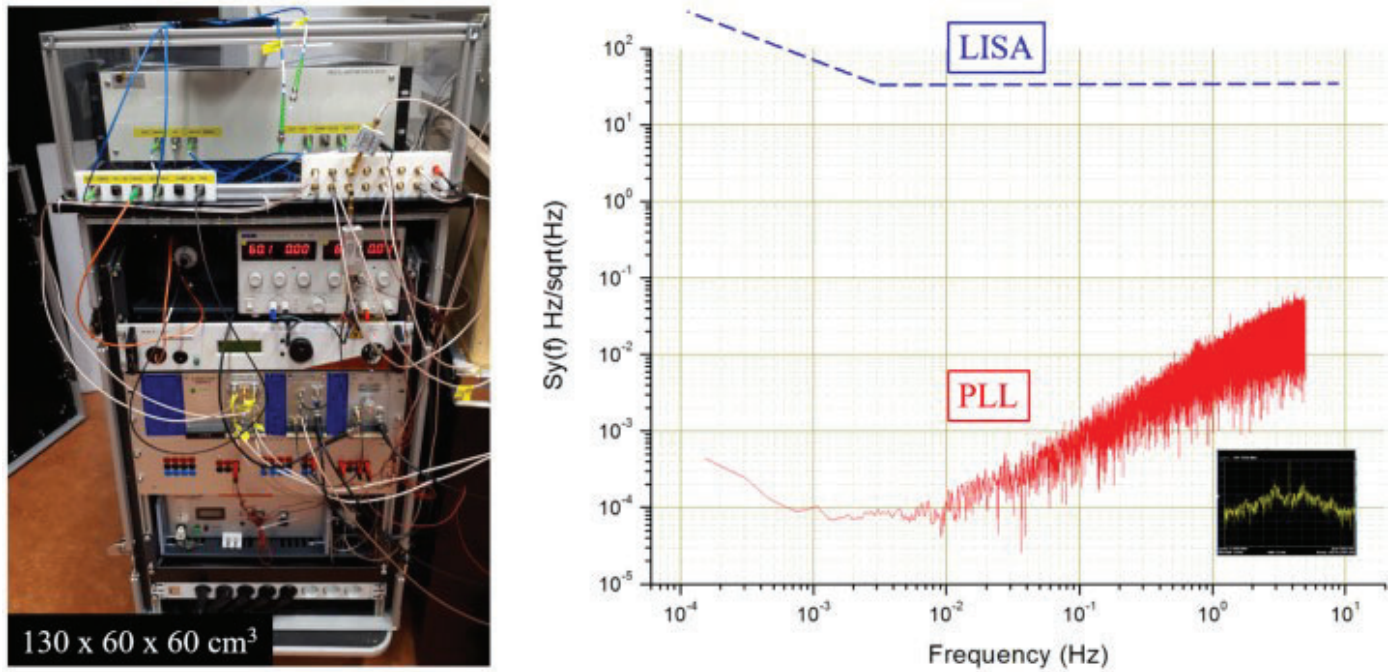

Figure 2. Left: Overview of the $1064 \mathrm{~nm}$ phase locked laser setup.

Right: Residual frequency noise of the PLL. Black insert shows the PLL frequency bandwidth $>34 \mathrm{kHz}$ 
The second part of the laser ensemble involves a frequency tripled Telecom laser, frequency stabilized to a hyperfine iodine line around $532.245 \mathrm{~nm}$, which corresponds to the second harmonic of the LISA mission nominal wavelength. This laser setup is totally fibered and will serve as a stable frequency reference for the tandem of $1064 \mathrm{~nm}$ phase locked lasers, while the iodine Doppler free spectroscopy is designed as a monolithic optical bench and will not require any realignment when transported to different sites. The frequency stabilization of the Telecom laser against iodine line, benefits from two previous experiments developed at SYRTE at $1542 \mathrm{~nm}$ and $1544 \mathrm{~nm}$. In both cases the residual frequency noise already achieved for this experiment is below the LISA mission requirements. We achieve a reproducible frequency stability below $2.5 \times 10^{-14} \tau^{-1 / 2}$ decreasing to $3.5 \times 10^{-15}$ level after $200 \mathrm{~s}$ of integration time. In terms of amplitude spectral density that corresponds to $15 \mathrm{~Hz} / \sqrt{ } \mathrm{Hz}$ (Figure 3 ).
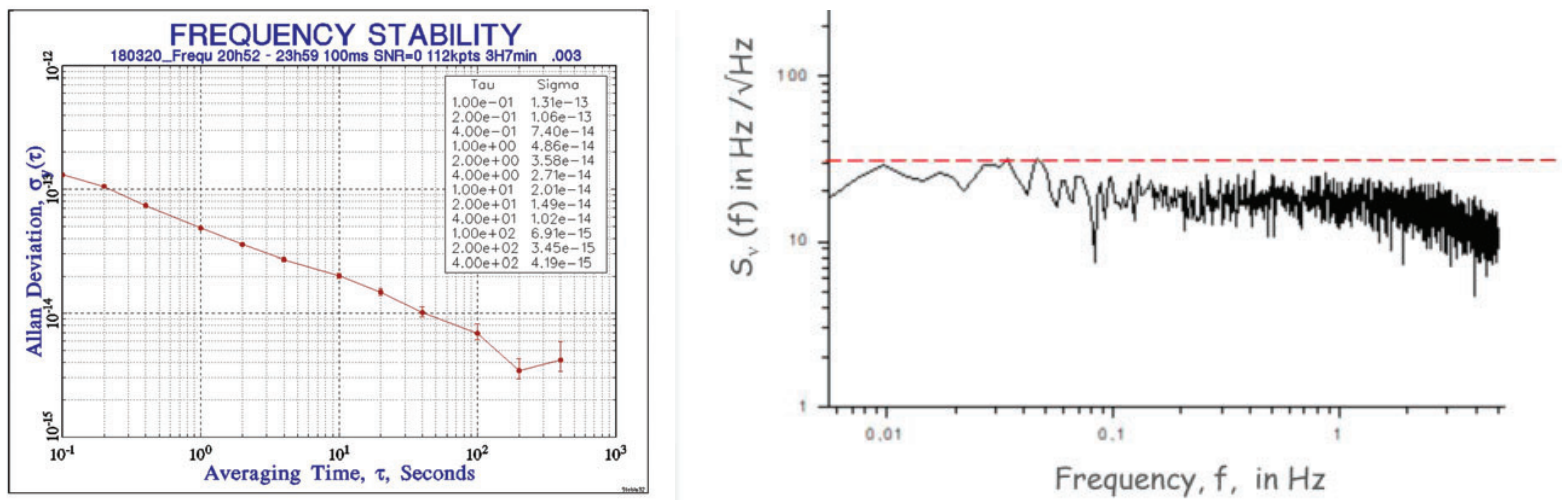

Figure 3. Left: Allan deviation of the fractional frequency instabilities of the iodine stabilized Telecom laser. Right: Amplitude spectral density of the residual frequency fluctuations of the iodine stabilized Telecom laser

The experimental setup used to fulfill the results shown above is based on fully fibered frequency tripled laser system, associated to iodine Doppler free spectroscopy operating in free space configuration (see Fig. 4) [6, 7]. The phase modulation at $220 \mathrm{kHz}$ is applied on the pump beam in the visible. The iodine cell operates at room temperature, surrounded only by thermal and magnetic shields.

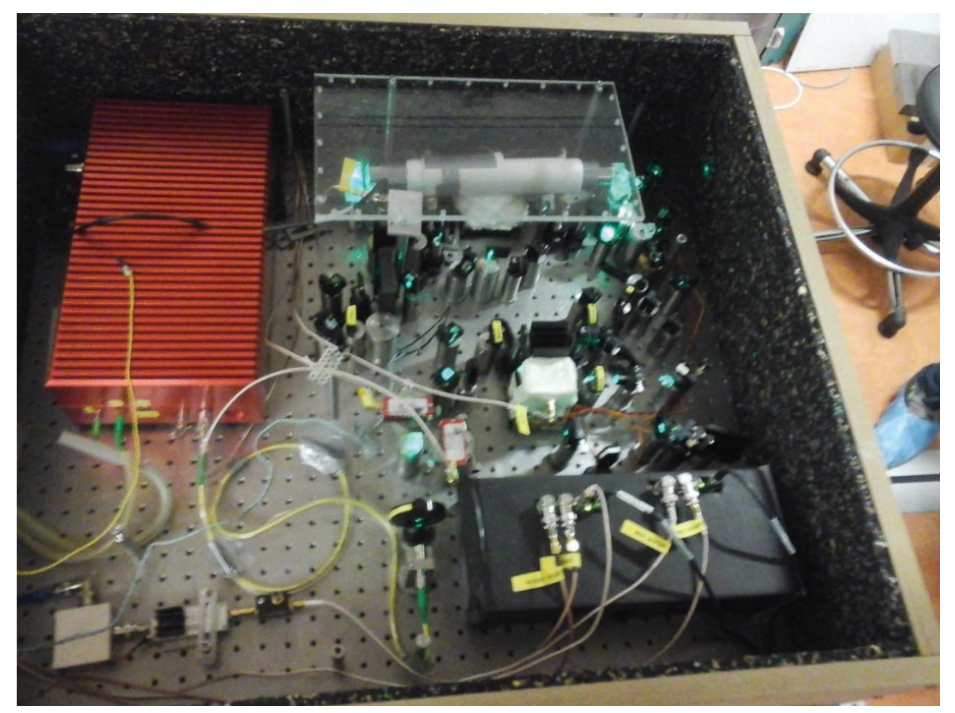

Figure 4. Photograph of the whole optical setup. The EDFA and the frequency tripling crystals are integrated in the red box at the left (Volume $\approx 51$ ). The dimensions of the spectroscopy optical bench are $40 \mathrm{~cm} \times 40 \mathrm{~cm}$. The cooled cell is visible at the top below a Plexiglass plate. At the bottom, the photodiodes are housed in a black box to avoid stray lights. The EOM is inserted in a homemade oven (white) for the temperature regulation. 


\section{EXPERIMENTAL SETUP}

\subsection{Design of the frequency tripled laser system}

As this laser device must be used on different sites of the partners forming the LISA France consortium, it is important that it can be easily carried, immune to any optical misalignment and should not require major interventions for its operation after a relocation. All Telecom components involved in this development are coupled by polarizationmaintaining optical fibers. They are highly reliable and commercially available, except the frequency sum generation crystal and the monolithic Doppler free iodine spectroscopy bench which are developed specifically for this project.

The fiber laser delivers $10 \mathrm{~mW}$ output power at $1596.7 \mathrm{~nm}$. Two Erbium doped optical amplifiers (EDFA) are used to fulfill the second harmonic generation (SHG: $\omega+\omega \rightarrow 2 \omega)$ and sum frequency generation $($ SFG: $\omega+2 \omega \rightarrow 3 \omega)$ processes. The frequency doubling crystal is a Lithium Niobate periodically poled (PPLN) waveguide crystal provided by NEL Company (Japan). Similar devices already used at SYRTE for use at different wavelengths (1542 nm and 1544 $\mathrm{nm}$ ) is described elsewhere [7]. For this project we generate $220 \mathrm{~mW}$ of harmonic power (798 nm) using $500 \mathrm{~mW}$ at the fundamental wavelength.

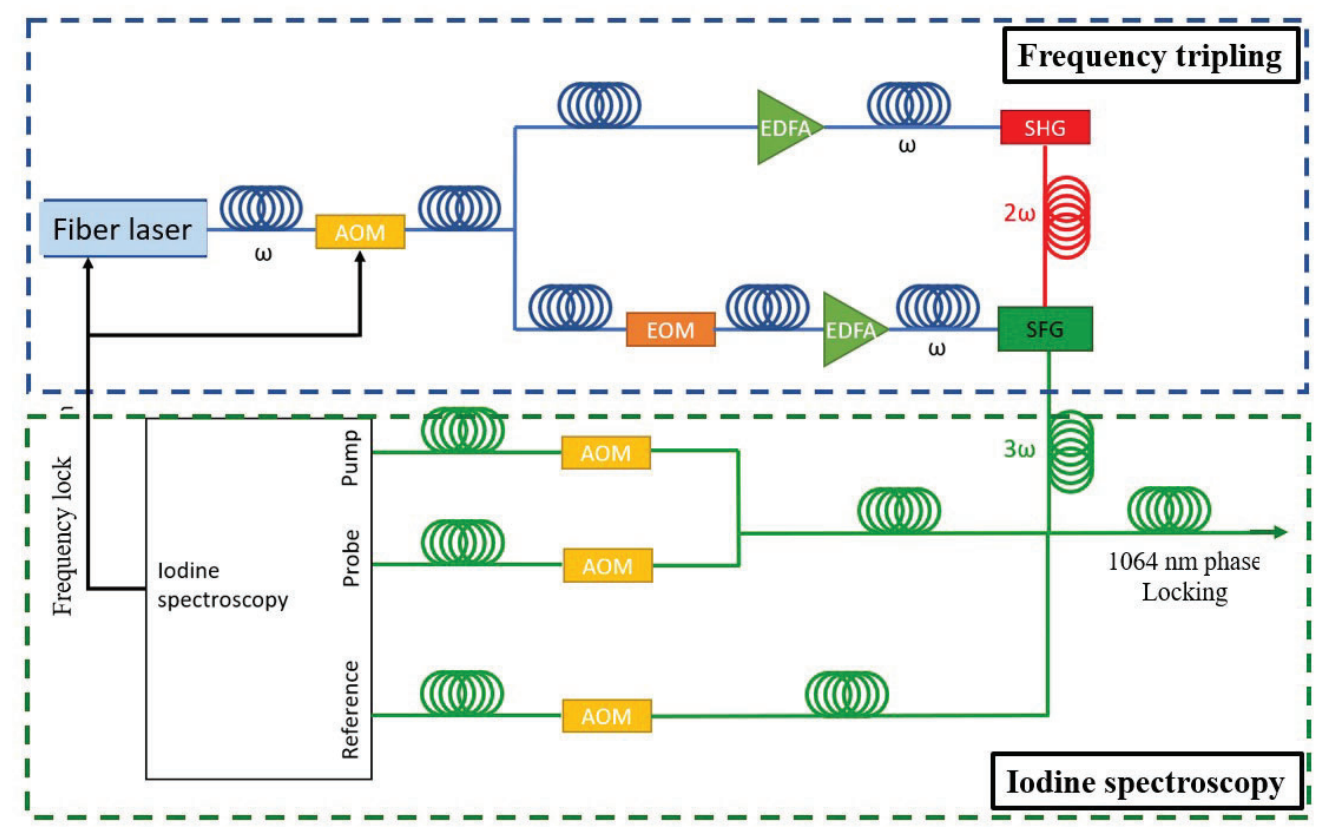

Figure 5. Schematic diagram of the laser setup. Top: laser unit including frequency tripling, modulation and frequency control. Bottom: Doppler free iodine spectroscopy

The SFG device is specifically developed for this project and uses à PPLN crystal from Covesion (UK). The optical arrangement, the fiber coupling as well as the packaging (see Fig. 6) is realized by Kylia (Fr). The PPLN is a Mg:O doped PPLN crystal $\left(40 \times 1 \times 0.5 \mathrm{~mm}^{3}\right)$ provided by Covesion Co (UK). The grating period is $7.30 \mu \mathrm{m}$, yielding to a phase matching temperature of $47^{\circ} \mathrm{C}$ for the $532 \mathrm{~nm}$ green radiation generation. A Peltier device combined with a specifically made oven is used to stabilize the crystal temperature within less $1 \mathrm{mK}$ of residual temperature fluctuations. 


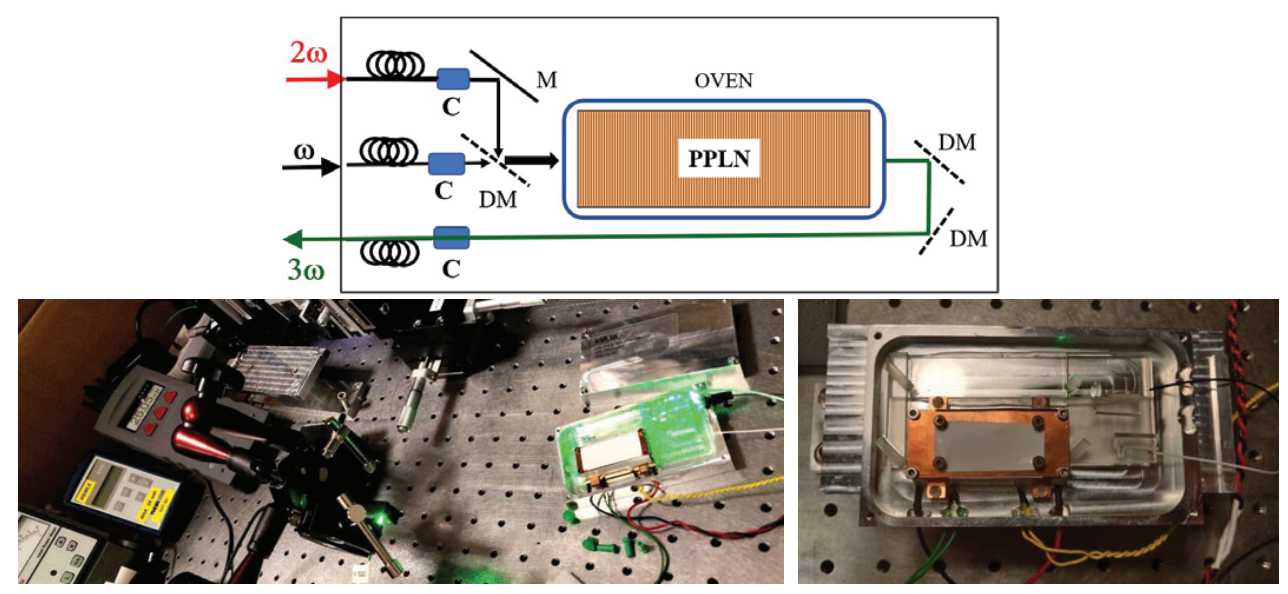

Figure 6. Top: SFG optical arrangement. C: collimators. M: mirror. DM: Dichroic filters. Bottom: pictures of the SFG module

A harmonic power of $38 \mathrm{~mW}$ at $532 \mathrm{~nm}$ is generated using $\mathrm{P}_{\omega}=1 \mathrm{~W}$ of infrared power associated to $\mathrm{P}_{2 \omega}=180 \mathrm{~mW}$ at $798 \mathrm{~nm}$. That corresponds to a nonlinear efficiency $\eta=\mathrm{P}_{3 \omega} / \mathrm{L}^{*}\left(\mathrm{P}_{\omega} * \mathrm{P}_{2 \omega}\right) \sim 5.5 \mathrm{~W}^{-1} \mathrm{~cm}^{-1}$, where $\mathrm{L}=4 \mathrm{~cm}$ is the length of the PPLN crystal. The final output power emerging at the output of the PM fiber, is $26 \mathrm{~mW}$. The optical losses are due to the use of two dichroic mirrors and the coupling in the PM fiber. The phase matching temperature acceptance is measured to be $\sim 0.8{ }^{\circ} \mathrm{C}$ at $47^{\circ} \mathrm{C}$.
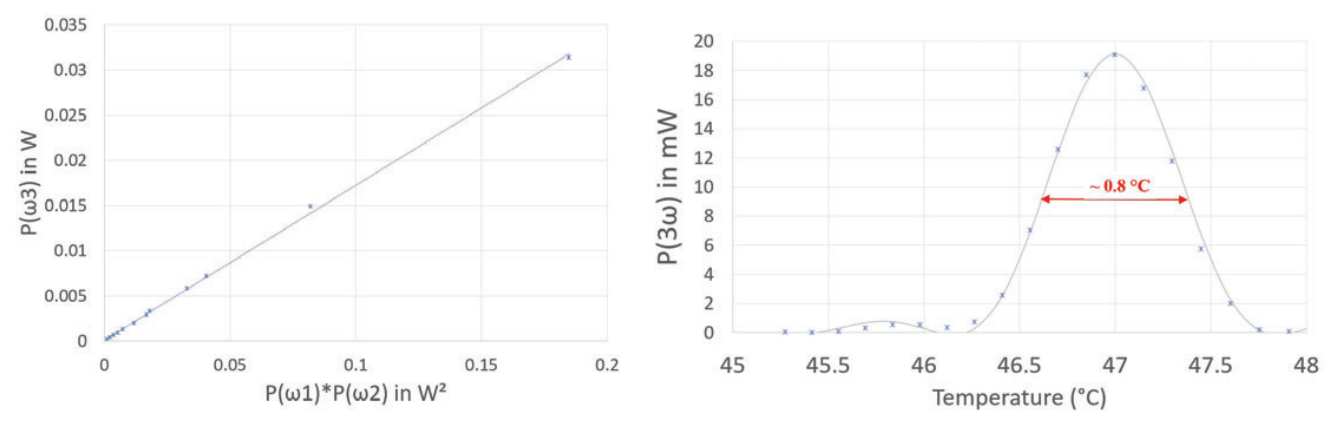

Figure 7. Performances of the Sum Frequency Generation device. Left: the power output at $532 \mathrm{~nm}$ in regards to the power inputs, The conversion efficiency. Right: the temperature phase matching condition for an infrared wavelength of $1596.6 \mathrm{~nm}$.
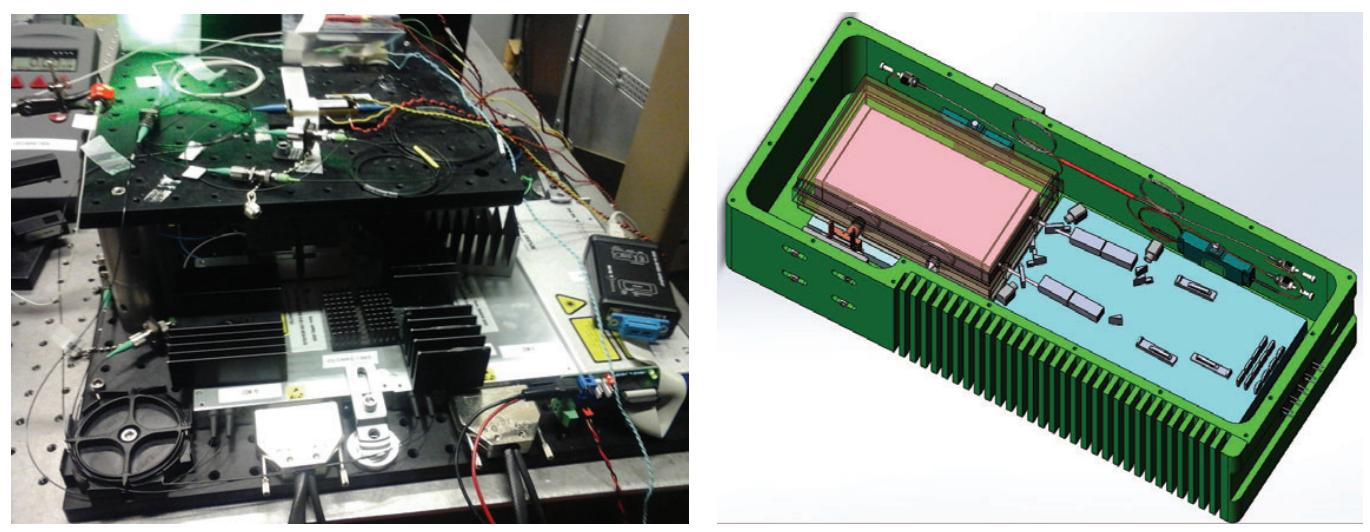

Figure 8. Overview of the laser setup under development. Left: frequency tripled laser device. Right: Iodine spectroscopy optical bench. 


\subsection{Design of the iodine Doppler free spectroscopy optical bench}

As previously mentioned, most of the components used in our experimental setup are commercially available, hence exhibiting a high TRL, except for the SFG and iodine spectroscopy assembly. To reach a high TRL for the whole laser setup (mandatory in order to be transportable and usable without requirement of any optical adjustments), we focused on the development of robust, compact and reliable optical assemblies of these two parts of the laser setup. To do so, we took advantage of Kylia free space optical assembly technology based on UV epoxies, which has been Telcordia qualified (Telcordia GR-1221). This include, but is not limited to, thermal cycling $\left(-40^{\circ} \mathrm{C} /+80^{\circ} \mathrm{C}\right)$, mechanical shocks $(500 \mathrm{G}, 1 \mathrm{~ms})$ and vibrations $(20 \mathrm{G}, 20-2000 \mathrm{~Hz})$. For a few years, Kylia assembly technology has also been qualified for space applications, with stronger environmental requirements (shock tests up to $1200 \mathrm{G}, 4000 \mathrm{~Hz}$ ). Therefore, we are confident that the devices developed for the laser experimental setup will easily reach a high TRL, enabling the whole system to meet the expected specifications.

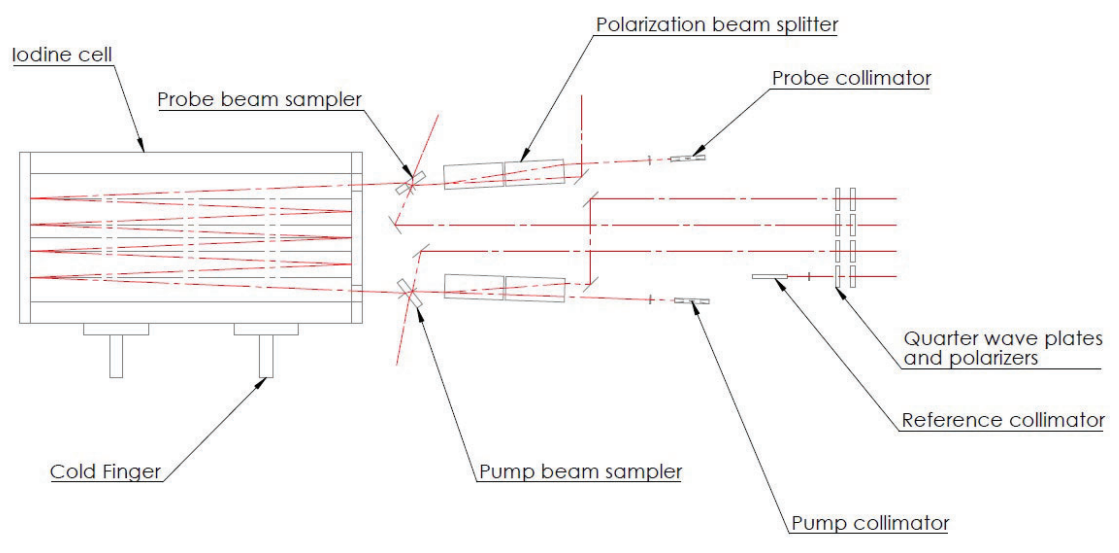

Figure 9. Schematic of the monolithic iodine Doppler free spectroscopy optical bench. On the left is the iodine cell, and on the right the optical inputs and outputs. The outgoing beams not used are to be dumped in light traps.

Using Kylia's know-how, a monolithic setup is made using a space-qualified single component epoxy to bond the iodine cell and the components to the fused silica substrate. The frequency feedback loop relies on a balanced optical detection of the probe beam. Two acousto-optical modulators are used to stabilize the pump and probe optical powers injected into the iodine cell. The beams are collimated and superimposed from the fibered output of the AOMs using extremely high precision techniques. Those techniques allow a rigorous waists and wave vectors superposition of the pump and the probe. All components are made of fused silica, including the mechanical mounts, and excepting the polarization beam separators, which are birefringent elements (Calcite). This allows to have the same thermal expansion coefficient $\left(5^{*} 10^{-}\right.$ $7^{7}{ }^{\circ} \mathrm{C}$ ) over the whole bench. Surfaces are AR coated, except for the beam samplers used for power stability. The schematic of optical setup is detailed in Fig. 9.

The iodine cell (provided by the Institute of Science Instrument (ISI / BRNO, CZ) has internal coating to provide the best reflections and the minimum power losses throughout the cell. It is used in an eight-pass optical configuration. The thermal regulation (around $-15^{\circ} \mathrm{C}$ ) is cooled through passive thermal management to reduce mechanical and magnetic noise inside the setup. The cell is surrounded by a twice magnetic shielding to reduce Zeeman's effect on the center line of the iodine hyperfine line [9]. The sensitivity is measured as $2 \times 10^{-15} / \mathrm{mG}$, for external magnetic fields below 2 Gauss. In our case, the measured attenuation factor of external fields is $>2000$.

Calcite crystals have been chosen as polarization beam splitters to reduce thermal dependencies and to reach a better polarization extinction ratio in comparison with other means. Over the length of the crystals about $20 \%$ of the power is absorbed. The collimators are specifically made to ensure waists overlapping at the center of the cell. The diameters are to be of 2.5 and $2.7 \mathrm{~mm}$ for the probe and the pump, respectively. The interaction length inside is 1.2 meters. Those parameters are known to allow an Allan deviation of $2 * 10^{-14} / \sqrt{ } \mathrm{Hz}[\mathrm{ref}]$. All the detectors are tilted and have a quarter wave plate with a polarizer in front of it to avoid feedback. The beam sampler has a $0.5^{\circ}$ wedge to avoid ghosting, and the stray light is managed through light traps and isolators. 


\section{CONCLUSION}

We presented the ongoing development of an ultra-stable, compact, and transportable laser setup to be used for the ground tests of the payload of the LISA mission. This setup is based on a Telecom laser emitting at $1596 \mathrm{~nm}$ frequency tripled and locked to an iodine line around $532 \mathrm{~nm}$. This development is based on previous successful work developed at SYRTE for frequency stabilization of Telecom lasers against iodine hyperfine lines around $514 \mathrm{~nm}$ and aims to reach a high TRL for the whole laser system. Optical subcomponents and processes used in this development are reproducible and will allow the possibility to duplicate the setup without any loss of performances. This will pave the way to the development of an industrial solution for demanding applications where high laser frequency stability is required. The laser setup will be used for accurate testing of interferometric benches mechanical stability needed for the MOSA-LISA AIVT, with the same level -or better- than the LISA mission requirements in terms of residual frequency instabilities.

This work has been performed with the support of LISA France, gathering 15 research laboratories and CNES, the French space agency. It was also supported by CNES (R\&T / R-S17/SU-0001), GRAM (Gravitation, Références, Astronomie, and Metrologie) and PhyFOG (Physique fondamentale et ondes gravitationnelles).

\section{REFERENCES}

[1] P.L. Bender, J.L. Hall, J. Ye, W.M. Klipstein, "Satellite-satellite laser links for future gravity missions", Space Science Reviews 108: 377-384, 2003

[2] T. Schuldt et al., "An ultra-stable frequency reference for space", ICSO 2014, International Conference on Space, Optics, Tenerife, Canary Islands, Spain, 7-10 October (2014)

[3] T. Leveque, C. Fallet, M. Mandea, R. Biancale, J. M. Lemoine, S. Tardivel, S. Delavault, A. Piquereau, Bourgogne, F. Pereira Dos Santos, R. Battelier, Ph. Bouyer, "Gravity Field Mapping Using Laser Coupled Quantum Accelerometers in Space, arXiv.org > physics > arXiv:2011.03382v1.

[4] J. Ye, L.-S. Ma, and J. L. Hall: Molecular iodine clock, Phys. Rev. Letters, vol. 87 (2001), p. 270801.

[5] E. J. Zang, J. P. Ciao, C. Y. Li, Y. K. Deng, and C. Q. Gao: "Realization of Four-Pass I2 Absorption Cell in 532-nm Optical Frequency Standard", IEEE Transactions on Instruments and Measurement, vol. 56 (2007), no. 2, pp. 673-676.

[6] C. Philippe et al., "Frequency tripled $1.5 \mu \mathrm{m}$ telecom laser diode, stabilized to iodine hyperfine line in the 1015 range" $30^{\text {th }}$ European Frequency and Time Forum, EFTF 2016, 4th-7th April 2016, York, United Kingdom. And references therein.

[7] C. Philippe et al.,"Efficient third harmonic generation of a CW-fibered $1.5 \mu \mathrm{m}$ laser diode, Appl. Phys. B (2016) 122, p. 265.

[8] N. Chiodo, F. Du-Burck, J. Hrabina, M. Lours, E. Chea, and O. Acef, "Optical phase locking of two infrared continuous wave lasers separated by 100 THz", OPTICS LETTERS / Vol. 39, No. 10 / May 15, 2014

[9] J. Barbarat et al. "Linear Zeeman Effect on Iodine-Based Frequency Stabilized Laser", Proceedings of IFCSEFTF'2019. Orlando, Florida (USA) 